\title{
Subject-Exoskeleton Contact Model Calibration Leads to Accurate Interaction Force Predictions
}

\author{
Gil Serrancoli $^{\circledR}$, Antoine Falisse ${ }^{\circledR}$, Christopher Dembia, Jonas Vantilt ${ }^{\circledR}$, Kevin Tanghe ${ }^{\circledR}$, \\ Dirk Lefeber, Ilse Jonkers, Joris De Schutter, and Friedl De Groote
}

\begin{abstract}
Knowledge of human-exoskeleton interaction forces is crucial to assess user comfort and effectiveness of the interaction. The subject-exoskeleton collaborative movement and its interaction forces can be predicted in silico using computational modeling techniques. We developed an optimal control framework that consisted of three phases. First, the foot-ground (Phase A) and the subjectexoskeleton (Phase B) contact models were calibrated using three experimental sit-to-stand trials. Then, the collaborative movement and the subject-exoskeleton interaction forces, of six different sit-to-stand trials were predicted (Phase $\mathrm{C}$ ). The results show that the contact models were able to reproduce experimental kinematics of calibration trials (mean root mean square differences (RMSD) coordinates $\leq 1.1^{\circ}$ and velocities $\left.\leq 6.8^{\circ} / \mathrm{s}\right)$, ground reaction forces (mean RMSD $\leq 22.9 \mathrm{~N}$ ), as well as the interaction forces at the pelvis, thigh, and shank (mean RMSD $\leq 5.4 \mathrm{~N}$ ). Phase $\mathbf{C}$ could predict the collaborative movements of prediction trials (mean RMSD coordinates $\leq 3.5^{\circ}$ and velocities $\leq 15.0 \% / \mathrm{s}$ ), and their subject-exoskeleton interaction forces (mean RMSD $\leq 13.1 \mathrm{~N}$ ). In conclusion, this optimal control framework could be used while designing exoskeletons to have in silico knowledge of new optimal movements and their interaction forces.
\end{abstract}

Index Terms-Movement prediction, exoskeleton, contact forces, dynamic optimization.

Manuscript received January 11, 2019; revised May 15, 2019; accepted June 18, 2019. This work was supported by the Agency Flanders Innovation and Entrepreneurship (VLAIO) under Grant MIRAD and Grant IWT-SBO 120057. The work of A. Falisse was supported in part by the Research Foundation Flanders (FWO) under Ph.D. Grant 1S35416N (Corresponding author: Gil Serrancolí.)

G. Serrancoli was with the Department of Mechanical Engineering, KU Leuven, B-3001 Leuven, Belgium. He is now with the Department of Mechanical Engineering, Universitat Politècnica de Catalunya, 08019 Barcelona, Spain (e-mail: gil.serrancoli@ upc.edu).

A. Falisse, I. Jonkers, and F. De Groote are with the Department of Movement Sciences, KU Leuven, 3001 Leuven, Belgium (e-mail: antoine.falisse@kuleuven.be; ilse.jonkers@kuleuven.be; friedl.degroote@ @uleuven.be).

C. Dembia is with the Department of Mechanical Engineering, Clark Center, Stanford University, Stanford, CA 94305 USA (e-mail: dembia@stanford.edu).

J. Vantilt, K. Tanghe, and J. De Schutter are with the Robotics Research Group, Department of Mechanical Engineering, KU Leuven, B-3001 Leuven, Belgium (e-mail: joris.deschutter@kuleuven.be).

D. Lefeber is with the R\&MM Research Group, Department of Mechanical Engineering, Vrije Universiteit Brussel, B-1050 Brussels, Belgium (e-mail: dlefeber@vub.ac.be).

Digital Object Identifier 10.1109/TNSRE.2019.2924536

\section{INTRODUCTION}

$\mathbf{O}$ VER the last twenty years, different types of exoskeletons have been designed and their wearability has been improved. However, obtaining user comfort and a safe cooperation between exoskeleton and user is still challenging [1], [2]. Both safety and comfort are related to interaction loads [3]. These loads can produce high pressures between the bony prominences and the device, which are the main cause of pressure ulcers [4]. The knowledge of the magnitude of these interaction forces and pressures during the design process of an exoskeleton would be crucial, since the design of exoskeletons could be adapted to avoid high pressures due to misalignments [5] and rigidity of the subject-device interface [6], which are common issues in exoskeleton designs. However, experimental values of these forces are usually not known in advance.

Computational modeling techniques could be used to estimate the interactions between a subject and an exoskeleton while building a physical prototype. However, the accurate prediction of the human-exoskeleton contact interactions and collaborative movement of the subject wearing the exoskeleton are still a challenge. This is mainly due to the dynamics redundancy (different combinations of forces can lead to the same kinematics), which makes it difficult to accurately estimate all involved forces and the kinematics simultaneously. Thus, the validation of computational models is crucial for trusting the results of these simulations [7].

There are some studies in the literature that attempted to optimize the movement of a subject wearing an exoskeleton with the goal of obtaining optimal controller designs of exoskeletons and improve their efficiency. Zhang et al. [8] optimized the assistance of an ankle exoskeleton experimentally to minimize the human energy of walking. Millard et al. [9] predicted the collaborative subjectexoskeleton movement of lifting a box solving an optimal control problem and coupling the subject and device with kinematic constraints. Manns et al. [10] optimized the parameters of a back exoskeleton modeled as a torsional spring for the prediction of the subject-exoskeleton collaborative movement of lifting a box. Apart from simulation, some experimental studies used sensors to measure interface pressures between a subject and an exoskeleton [11], [12]. 
However, as far as the authors know, no simulation study has yet rigorously validated or predicted human-exoskeleton contact forces. The novel contribution of this study is the calibration of both foot-ground and subject-exoskeleton compliant contact models using experimental data of sit-to-stand trials for one subject wearing a bilateral lower-limb exoskeleton, and the prediction of collaborative movement and its interaction forces for a separate set of sit-to-stand trials. This framework is intended to be the basis for simulating new optimal movements and their realistic forces while building exoskeletons. We hypothesize that we can accurately describe resultant human-exoskeleton interaction forces and collaborative human-exoskeleton movements using a simple, wellcalibrated contact model.

\section{Methodology}

\section{A. Experimental Measurements}

Kinematic and dynamic data of a healthy 29 year-old subject (gender: male, mass: $70 \mathrm{~kg}$ ) wearing a bilateral exoskeleton were recorded during sit-to-stand movements. The bilateral exoskeleton was actuated at the ankle, knee, and hip joints with the purpose of assisting subjects with muscle deficiency during sit-to-stand movements [13], [14]. Contact pressures were measured at the contact zones between the subject and exoskeleton. These contact surfaces were at the pelvis through a module covering the circumference of the pelvis, and two commercial braces at the thigh and shank linked to the structure of the exoskeleton.

Kinematic data were obtained at $100 \mathrm{~Hz}$ from trajectories of 56 markers attached to the human (Vicon Motion Systems, Oxford, UK) and they were low-pass filtered with a Butterworth filter at $6 \mathrm{~Hz}$. Foot-ground and chair-ground contact forces were measured by three force plates (AMTI, Watertown, MA) at $1000 \mathrm{~Hz}$, and low-pass filtered at $6 \mathrm{~Hz}$. Exoskeleton joint angles were obtained from encoders at the joints and exoskeleton joint moments were estimated as a function of joint angles (also low-pass filtered at $6 \mathrm{~Hz}$ ) and previously-identified dynamic parameters [15], [16].

Subject-exoskeleton interface pressures were measured at $50 \mathrm{~Hz}$ with two matrices of capacitive sensors (matrices of $16 \mathrm{x}$ 8 sensors and $32 \times 8$ sensors, with $2 \mathrm{~cm}^{2}$ each sensor, S2140 and S2154, Novel, Munich, Germany) attached to the body of the subject. These data were low-pass filtered using a Butterworth filter at $6 \mathrm{~Hz}$. Two configurations were tested, one with both sensor matrices covering the whole interface between the subject and the pelvis module, and the other with one sensor matrix covering the interface area at the thigh and the other one covering the shank region (Fig. 1 right).

For each sensor matrix configuration, we captured 3D scans (Artec, Luxembourg, Luxembourg) of the subject with the sensors to know their location with respect to the segments of the body. We used the subject-specific geometry of the body to map the pressure values of the sensor matrix to the surface points of the subject. The resultant contact force vector was calculated at each frame by multiplying the pressure values by the covered area.

Five trials of three sit-to-stand movements were captured for each sensor configuration, with the exoskeleton providing

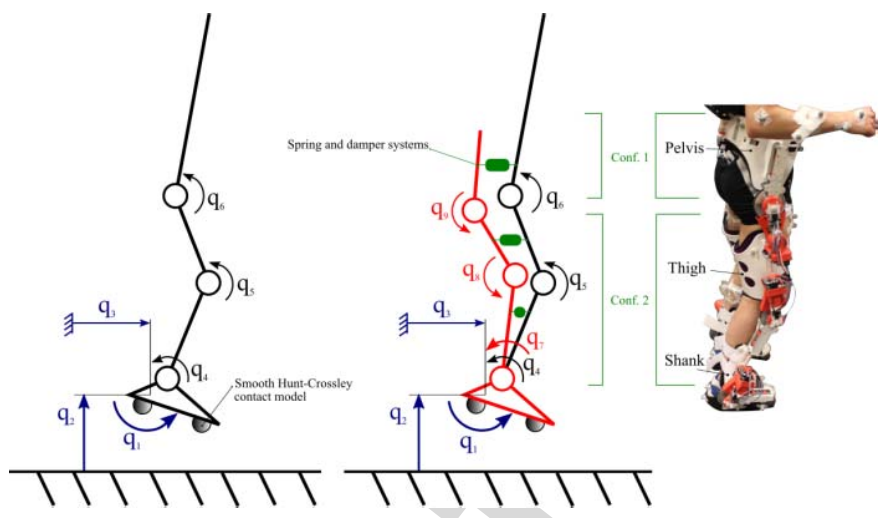

Fig. 1. Left: initial model to calculate human joint resultant moments with six DOFs. Middle: model to simulate the collaborative movement with nine DOFs. Right: picture of the subject wearing the exoskeleton. $q_{1}, q_{2}$ and $q_{3}$ : DOFs of the foot with respect to ground; $q_{4}, q_{5}$ and $q_{6}$ : relative DOFs of the human; $q_{7}, q_{8}$ and $q_{9}$ : relative DOFs of the exoskeleton. Conf. 1 and 2 indicate the locations where we had experimental contact forces.

sit-to-stand assistance (active mode) and with the exoskeleton unpowered (passive mode). In total, 60 movements were captured. Of those, 3 sit-to-stand trials were used to calibrate the contact models and 6 to predict the subject-exoskeleton movements. To become familiar with the exoskeleton, the subject performed three sit-to-stand movements with the exoskeleton before we recorded data. In this line, we used the last movement of the three latest trials. The study was approved by the ethical committee of KU Leuven and the subject signed a prior consent form.

\section{B. Description of the Model}

The human and exoskeleton were represented as a twolegged planar torque-driven model (foot, shank, thigh, and pelvis). The dominant dynamic moments and interaction forces in sit-to-stand movements are in the sagittal plane, therefore a planar model was used. First, a simplified version was used to compute the joint moments, and then a model with two kinematic chains (human and exoskeleton) was used to simulate the collaborative movement.

Because we initially had no information about human joint torques and contact forces dynamically consistent with the kinematics, first, we used a six-degree-of-freedom (DOF) model with the exoskeleton rigidly attached to the human and perfectly aligned (three DOFs between the foot and the ground, and one DOF at each of the ankle, knee, and hip joints) (Fig. 1 left). No markers were attached to the human pelvis, so we considered the pelvis to be aligned with the trunk, and markers on the trunk were used to capture the orientation of those bodies. Inverse kinematics analysis from marker data was carried out using this model in OpenSim [17] to obtain joint angles of the human and then inverse dynamics was performed to obtain the resultant (subject + exoskeleton) joint moments. To obtain the human joint moments, the experimentally measured exoskeleton joint moments were subtracted from the resultant ones calculated using OpenSim.

Second, a model with two kinematic chains was used to simulate the collaborative movement between the subject 

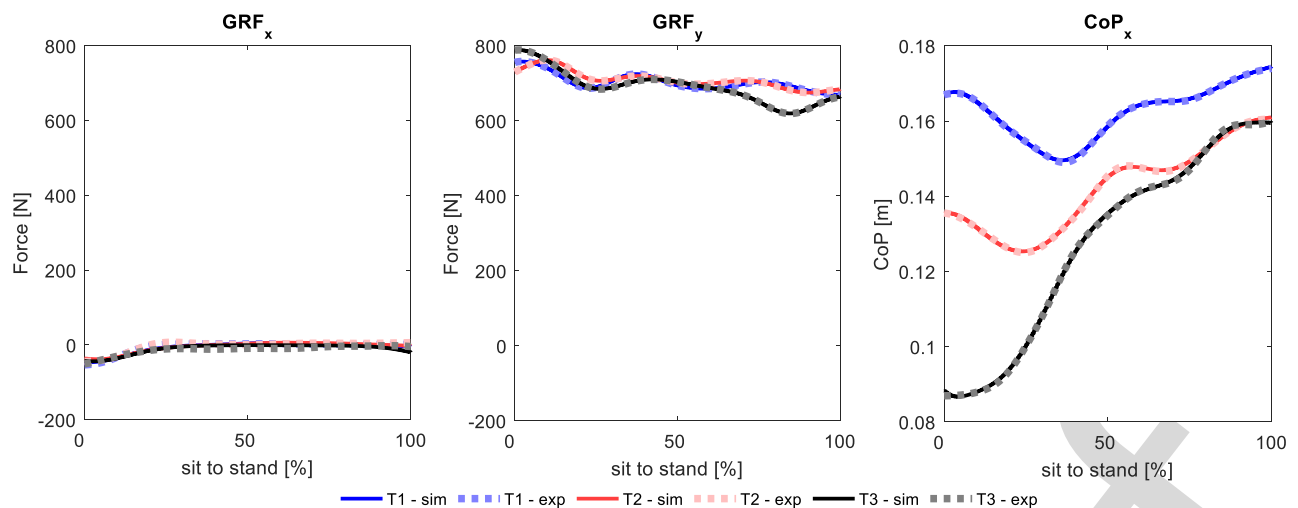

Fig. 2. Ground reaction forces from the calibration trials in Phase A. GRF $x$ and GRF $y$ are the horizontal and vertical components respectively, and $\mathrm{CoP}_{X}$ is the location of the center of pressure with respect to the lab reference. T1, T2 and T3 are the data for all three calibration trials. sim stands for simulated data and exp for experimental.

TABLE I

Design VARIABLES AND Cost Function TERmS

\begin{tabular}{|c|c|c|c|c|}
\hline & Phase & $\mathrm{A}$ & $\mathrm{B}$ & $\mathrm{C}$ \\
\hline \multirow{5}{*}{$\begin{array}{c}\text { Design } \\
\text { variables }\end{array}$} & Foot-ground contact parameters & $X$ & & \\
\hline & S-E contact parameters & & $X$ & \\
\hline & $\begin{array}{l}\text { States: } \\
\text { coordinates, velocities and } \\
\text { accelerations }\end{array}$ & $X$ & $X$ & $X$ \\
\hline & $\begin{array}{l}\text { Controls: } \\
\text { jerks and foot-ground reaction } \\
\text { forces }\end{array}$ & $X$ & $X$ & $X$ \\
\hline & Subject-exoskeleton forces & & $X$ & $X$ \\
\hline \multirow{8}{*}{$\begin{array}{l}\text { Weighted } \\
\text { cost } \\
\text { function } \\
\text { terms }\end{array}$} & Ground-reaction forces & $t$ & $t$ & $t$ \\
\hline & $\mathrm{CoP}$ & $t$ & $t$ & $t$ \\
\hline & Coordinates & $t$ & $t$ & \\
\hline & Velocities & $t$ & $t$ & \\
\hline & Joint torques & $m$ & $t$ & $t$ \\
\hline & Jerk controls & $m$ & $m$ & $m$ \\
\hline & S-E contact energy & & $m$ & $m$ \\
\hline & S-E contact forces & & $t$ & \\
\hline
\end{tabular}

$X$ stands for the corresponding variable being optimized during the phase, $t$ means that the corresponding time variable is tracked and $m$ that is minimized. Formulations for phases $\mathrm{C} 1$ and $\mathrm{C} 2$ are represented as $\mathrm{C}$.

and the exoskeleton (Phases A to C, see Optimization Formulations section). The human system consisted of a foot, shank, thigh, and pelvis, and had six DOFs (three DOFs between the foot and the ground, and one DOF at each of the ankle, knee, and hip joints). The exoskeleton system consisted of a foot-plate (rigidly attached to the human foot), shank, thigh, and pelvis segments. The exoskeleton ankle, knee, and hip joints were modeled as hinge joints (one DOF at each of the ankle, knee, and hip exoskeleton joints) (Fig. 1 right).

A smooth foot-ground Hunt-Crossley contact model was used to simulate the force between the exoskeleton and the ground. The contact was modeled between two spheres (one at the heel and one at the toes) and the ground plane. The original Hunt-Crossley contact model in Simbody [18] was smoothed (see Appendix 1). The subject-exoskeleton contact model consisted of three linear and rotational springand-damper systems, one in between each pair of bodies (bushing forces in OpenSim). This model represented the
TABLE ॥

RMSD BETWEEN MOdEL AND EXPERIMENTAL VARIABLES

\begin{tabular}{|c|c|c|c|}
\hline & Trial & Phase A & Phase B \\
\hline \multirow{3}{*}{$\begin{array}{c}\text { Coordinates } \\
\text { [degree] }\end{array}$} & Cal1 & $0.4 \pm 0.1$ & $1.0 \pm 0.4$ \\
\hline & $\mathrm{Ca} 2$ & $0.5 \pm 0.1$ & $0.7 \pm 0.3$ \\
\hline & $\mathrm{Cal} 3$ & $0.8 \pm 0.2$ & $1.1 \pm 0.4$ \\
\hline \multirow{3}{*}{$\begin{array}{l}\text { Velocities } \\
\text { [degree/s] }\end{array}$} & Call & $3.5 \pm 1.5$ & $6.1 \pm 2.6$ \\
\hline & $\mathrm{Cal} 2$ & $4.3 \pm 1.7$ & $5.4 \pm 2.2$ \\
\hline & $\mathrm{Cal3}$ & $6.5 \pm 1.8$ & $6.8 \pm 1.9$ \\
\hline \multirow{3}{*}{$\mathrm{GRF}_{\mathrm{x}}[\mathrm{N}]$} & Cal1 & 4.1 & 13.3 \\
\hline & $\mathrm{Ca} 2$ & 6.3 & 12.0 \\
\hline & $\mathrm{Cal} 3$ & 6.6 & 22.9 \\
\hline \multirow{3}{*}{$\mathrm{GRF}_{\mathrm{y}}[\mathrm{N}]$} & Call & 0.5 & 1.0 \\
\hline & $\mathrm{Ca} 2$ & 0.4 & 0.5 \\
\hline & $\mathrm{Cal} 3$ & 0.6 & 0.7 \\
\hline \multirow{3}{*}{$\mathrm{CoP}_{\mathrm{x}}[\mathrm{mm}]$} & Call & 0.2 & 0.8 \\
\hline & $\mathrm{Cal} 2$ & 0.3 & 0.9 \\
\hline & $\mathrm{Cal} 3$ & 0.4 & 3.1 \\
\hline \multirow{3}{*}{$\begin{array}{l}\text { Human joint } \\
\text { moments } \\
{[\mathrm{Nm}]}\end{array}$} & Cal1 & & $7.3 \pm 5.3$ \\
\hline & $\mathrm{Ca} 2$ & & $6.6 \pm 2.6$ \\
\hline & $\mathrm{Cal} 3$ & & $9.4 \pm 2.4$ \\
\hline \multirow{3}{*}{$\begin{array}{l}\text { Exoskeleton } \\
\text { joint moments } \\
{[\mathrm{Nm}]}\end{array}$} & Call & & $4.3 \pm 3.6$ \\
\hline & $\mathrm{Cal} 2$ & & $2.7 \pm 2.2$ \\
\hline & $\mathrm{Cal} 3$ & & $5.5 \pm 0.4$ \\
\hline \multirow{2}{*}{ Pelvis c.f. $[\mathrm{N}]$} & Call & & 5.4 \\
\hline & $\mathrm{Cal} 2$ & & 4.5 \\
\hline Thigh c.f. $[\mathrm{N}]$ & Cal3 & & 2.3 \\
\hline Shank c.f. [N] & $\mathrm{Cal3}$ & & 2.2 \\
\hline
\end{tabular}

$\mathrm{GRF}_{\mathrm{x}}$ and $\mathrm{GRF}_{\mathrm{y}}$ stand for horizontal and vertical ground reaction forces, $\mathrm{CoP}_{\mathrm{x}}$ for horizontal location of the centre of pressure, and $\mathrm{c}$. $\mathrm{f}$. for contact force. For joint coordinates, velocities and moments, mean \pm standard deviation across all joints are shown. Cal1, Cal2 and Cal3 stand for calibration trials 1, 2 and 3.

main stiffness and damping components of the interaction forces.

\section{Optimization Formulations}

Contact model parameters need to be calibrated in order to obtain realistic movement and force predictions. In this case, we calibrated both contact models first, and then we predicted the collaborative movement. The calibration process was split into two phases due to computational time and convergence reasons. The whole process consisted of three main phases: the calibration of the foot-ground contact parameter values (Phase A), the calibration of the humanexoskeleton contact parameter values (Phase B), and the prediction of the movement and its forces using the calibrated 

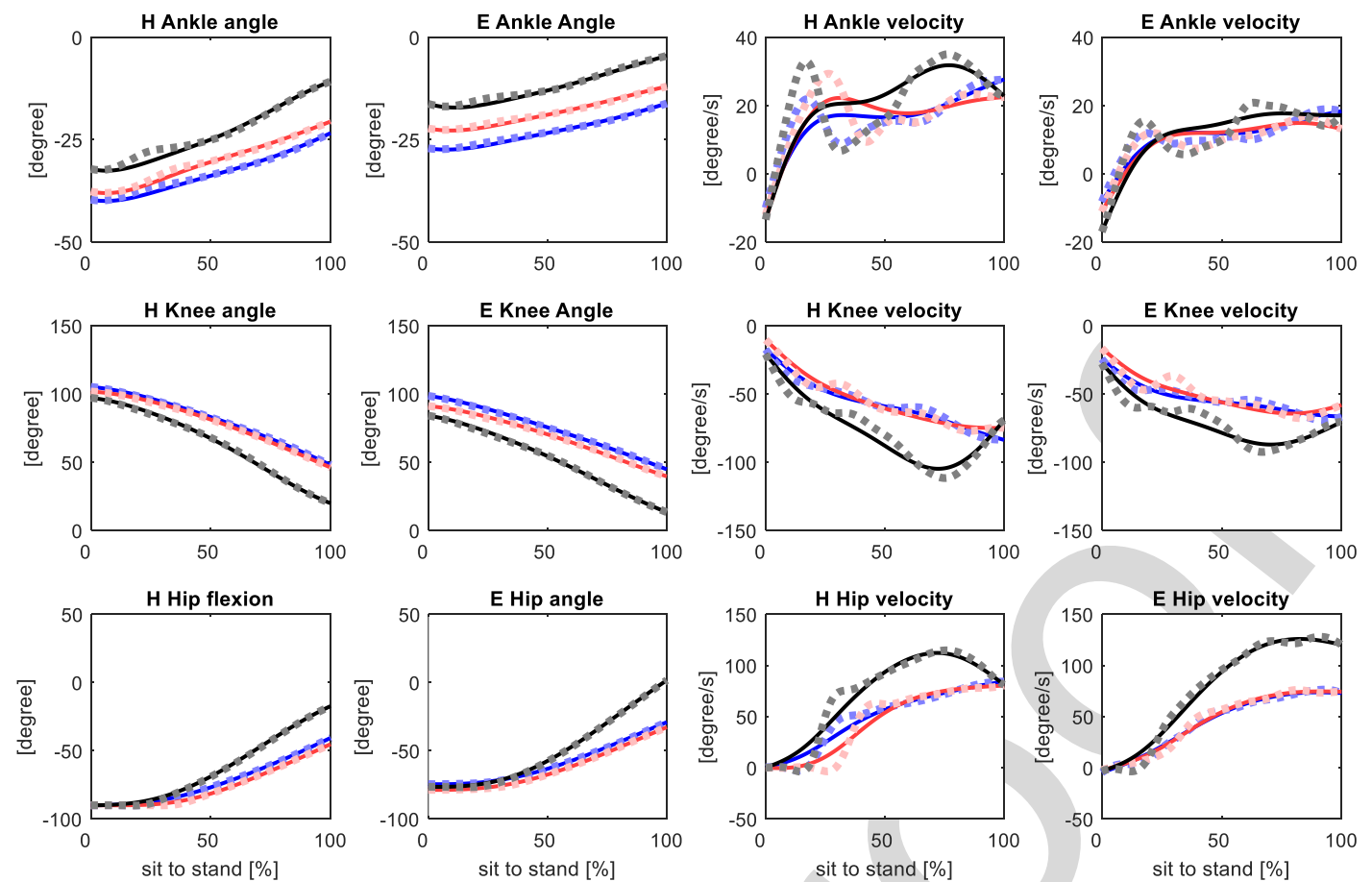

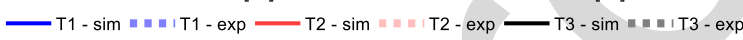

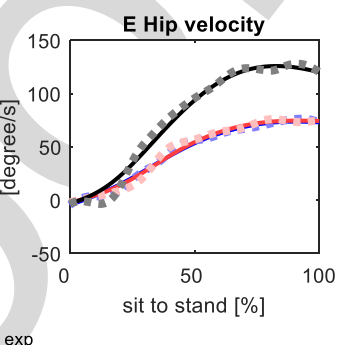

Fig. 3. Kinematics of the calibration trials in Phase A. Angles and angular velocities for ankle, knee and hip joint angles of the human $(\mathrm{H})$ and exoskeleton (E) side. T1, T2 and T3 are the data for all three calibration trials. sim stands for simulated data and exp for experimental.

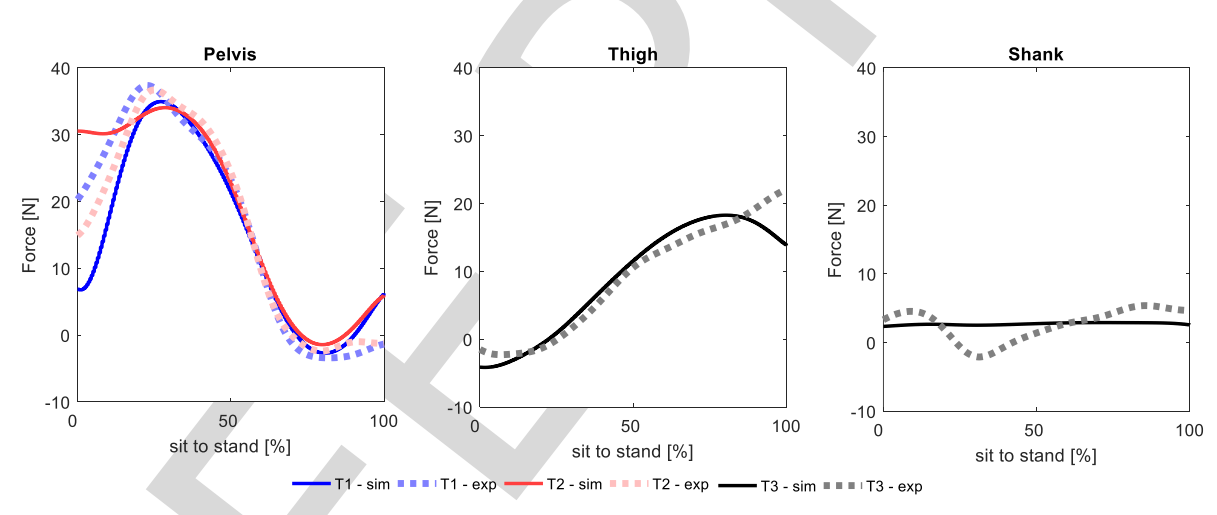

Fig. 4. Resultant subject-exoskeleton contact forces at the pelvis (left), thigh (middle) and shank (right) in Phase B. T1, T2 and T3 are the data for all three calibration trials. sim stands for simulated data and exp for experimental.

models (Phase C1). We also repeated Phase C1, perturbing the subject-exoskeleton parameter values (Phase $\mathrm{C} 2$ ). In all phases, an optimal control problem was formulated and solved using a direct collocation method to obtain the optimal state (coordinates, velocities, and accelerations in all phases), control and parameter values.

The time line was discretized with 200 nodes per second and 4 collocation points per interval and states were parameterized with $3^{\text {rd }}$ order Lagrange polynomials (pseudospectral approach). An implicit dynamic formulation was used, which implies that the equations of motion were enforced as algebraic constraints rather than as differential constraints at each time interval [19], and the jerks (derivative of accelerations) were included as controls. We calculated the residuals of the equations of motion using the API of OpenSim and Simbody. We also included constraints to ensure continuity of state variables between intervals and continuity of state derivatives (defect constraints) within each interval. The optimal control problems were solved using CasADi [20], a symbolic framework for algorithmic differentiation, from MATLAB, which relies on IPOPT [21] to solve the NLP (code in SimTK webpage: https://simtk.org/projects/predicsubjexosk).

Phase A:

In Phase A, the foot-ground contact parameter values were optimized so that they could reproduce experimental contact forces. The main parameters of the foot-ground contact model are the stiffness and damping properties, the location of the spheres with respect to the foot (local coordinates horizontal and vertical) and the radius of the spheres. We performed a parameter identification analysis to choose which parameters had the greatest influence on the contact forces (following the method of Van den Hof et al. [22]). 

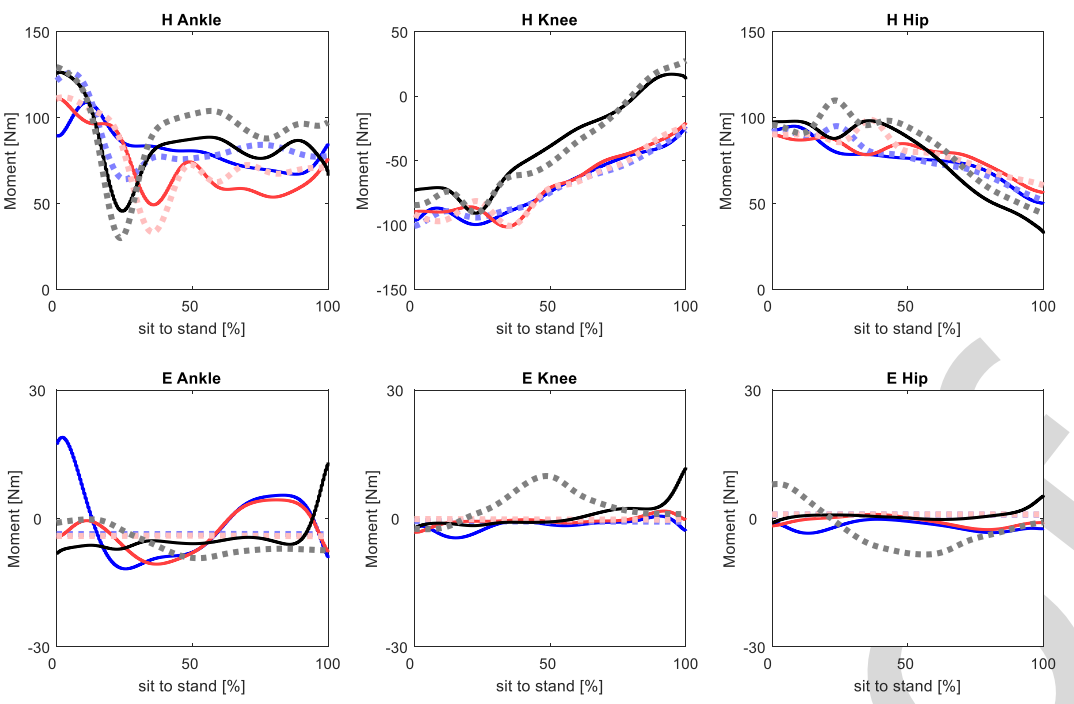

Fig. 5. Human (H) and exoskeleton (E) joint moments in Phase B. T1, T2 and T3 are the data for all three calibration trials. sim stands for simulated data and exp for experimental. Note that the scale of the plots for the human and exoskeleton joint moments is not the same.

We concluded that the radius of the spheres and the vertical coordinate of the location of the spheres were coupled. Therefore, we excluded the radius of the sphere from the group of optimization design variables.

In this phase, the optimal control problem consisted of estimating the foot-ground contact parameter values listed above, as well as the states and controls (joint torques, jerks and ground reaction forces) between an initial and a final state. One set of contact parameters was calibrated through the simultaneous use of three sit-to-stand movements with the exoskeleton in passive mode (calibration trials), to avoid trialspecific parameter values. Pelvis contact force was available in two of those three calibration trials, and thigh and shank contact forces were available for the third calibration trial. The cost functional included terms to track experimental joint angular coordinates and velocities, as well as ground reaction forces and the horizontal location of the center of pressure $(\mathrm{CoP})$ (Table I), and terms to minimize joint torques. Subject-exoskeleton interaction forces were considered null, assuming no contact between the subject and the exoskeleton at the shank, thigh, and pelvis. In this phase, we assumed that the joint torques will have the values needed to support the system. It is in phase B where we obtained a contact model able to reproduce experimental contact forces.

\section{Phase B}

In Phase B, we calibrated the parameter values of the spring and damper systems that model the contact between the subject and exoskeleton. In order to reduce the number of design variables, we excluded the damping parameters from the set of design variables. We assumed that for movements with small relative translations and velocities, the damping term could perform a similar effect as the stiffness term (due to the non-varying forces) and introduce redundancy in the optimization. Therefore, we set them to constant values (10 Ns/m for the translational damping and $0.1 \mathrm{Nms} / \mathrm{rad}$ for the rotational damping, similar to the contact parameters of a grip contact model [23]). Therefore, we selected as design variables the origin locations of the three spring and damper systems (with respect to the human body), and linear (different for tangential and normal directions of the human segments) and rotational stiffness.

The optimal control problem consisted of estimating the subject-exoskeleton contact parameters listed above, and the same state and control variables between the same given states as in the previous phase (calibration trials). The only difference was the addition of subject-exoskeleton contact forces as controls so that the optimizer had more flexibility. Foot-ground contact parameter values were set to the ones obtained in Phase A. We tracked the experimental angular coordinates and velocities, ground reaction forces, and the location of the CoP, as in Phase A. In addition, in Phase B we also tracked the experimental joint moments, and the component perpendicular to the interface surface of the resultant contact forces at the shank and thigh for one trial, and at the pelvis for two trials. As pressure sensors only measure normal force, not all components of the contact wrench are available. Therefore, we minimized the squared value of the contact energy of the contact wrench components, for which we did not have experimental data. See Table I for the summary of the design variables and cost function terms.

Phase C

In Phase $\mathrm{C} 1$, we used the calibrated foot-ground and subject-exoskeleton contact parameter values to predict sitto-stand movements (both kinematics and subject-exoskeleton contact forces) of six different trials with the exoskeleton providing assistance (prediction trials, three with information of experimental pressure data at the pelvis and three at the shank and thigh). In this case, we optimized states and controls (the same as in Phase B), but joint kinematics were not tracked, only the initial and final states were given. We tracked experimental ground reaction forces and the location of the CoP, and joint torques (Table I). We also minimized the squared value of 
the human-exoskeleton interaction energy for all components of the contact wrench to avoid redundancy in the optimization.

In Phase $\mathrm{C} 2$, we solved the same optimal control problem as in Phase $\mathrm{C} 1$, but multiplying the parameters of the subjectexoskeleton interaction forces by a factor of 1.4 (equivalent to the variability observed in the peak interaction forces for 9 subjects), to identify the influence of those parameters on the prediction of the collaborative movement and interaction forces.

Root mean squared differences (RMSD) between model and experimental variables were calculated in both calibration trials (Phase A and B) and prediction trials (Phase C1 and Phase $\mathrm{C} 2$ ). The computational time for solving each optimization problem was about 3 hours for Phases $\mathrm{A}$ and $\mathrm{B}$, and 30 minutes for Phases $\mathrm{C} 1$ and $\mathrm{C} 2$.

\section{Results}

\section{A. Phase A. Calibration of Foot-Ground Contact Model}

The results of Phase A show that the calibrated footground contact parameter values could accurately reproduce the experimental ground reaction forces (horizontal and vertical), and the distance to the $\mathrm{CoP}$ for all three calibration trials (Fig. 2). The root mean square differences (RMSD) were lower than $10 \mathrm{~N}$ for all forces and below $1.0 \mathrm{~mm}$ for the CoP (Table II). Joint angles and velocities were also tracked well (Fig. 3). The highest mean and standard deviations of RMSD (poorest estimation) across all calibration trials were $0.8 \pm$ 0.2 degrees for joint angles, and $6.5 \pm 1.8$ degrees/s for joint angular velocities (Table II).

\section{B. Phase B. Calibration of Subject-Exoskeleton Contact Model}

The optimized subject-exoskeleton contact model accurately reproduced the magnitude of subject-exoskeleton contact forces (Fig. 4), with RMSD values comparable to the tracking of ground reaction forces. In this phase, the highest RMSD were for tangential ground reaction forces with $22.9 \mathrm{~N}$, and with a mean RMSD for vertical and horizontal GRF over all calibration trials of $8.4 \mathrm{~N}$, whereas the highest RMSD for contact forces were at the pelvis with $5.4 \mathrm{~N}, 2.3 \mathrm{~N}$ for the thigh, and $2.2 \mathrm{~N}$ for the shank (Table II). The joint moments obtained in this phase were also accurate (mean RMSD < $10 \mathrm{Nm}$ ) (Fig. 5).

The tracking of kinematics was slightly worse than in Phase A. The highest mean and standard deviations of RMSD for joint angles were $1.1 \pm 0.4$ degrees, and for joint angular velocities $6.8 \pm 1.9$ degrees/s (Table II). RMSD for kinematics, GRF and subject-exoskeleton contact forces are lower than the maximum values of one standard deviation for six experimental trials.

\section{Phase C. Prediction of Collaborative Movement and Interaction Contact Forces}

The goal of this phase is to validate that the calibrated model is able to predict the kinematics and contact forces close to the experimental values. Calibrated contact models from Phases $\mathrm{A}$ and $\mathrm{B}$ were able to predict joint kinematics of prediction
TABLE III

RMSD BETWEen MOdEL AND EXPERIMENTAL VARIABLES

\begin{tabular}{|c|c|c|c|}
\hline & Trial & Phase C1 & Phase C2 \\
\hline \multirow{6}{*}{$\begin{array}{l}\text { Coordinates } \\
\text { [degree] }\end{array}$} & Pre1 & $1.0 \pm 0.7$ & $1.1 \pm 0.6$ \\
\hline & Pre2 & $1.3 \pm 0.8$ & $1.1 \pm 0.6$ \\
\hline & Pre3 & $1.0 \pm 0.3$ & $0.9 \pm 0.6$ \\
\hline & Pre4 & $3.3 \pm 2.0$ & $3.2 \pm 1.9$ \\
\hline & Pre5 & $2.0 \pm 1.1$ & $1.5 \pm 1.1$ \\
\hline & Pre6 & $3.2 \pm 1.8$ & $3.1 \pm 1.7$ \\
\hline \multirow{6}{*}{$\begin{array}{l}\text { Velocities } \\
{[\text { degree/s] }}\end{array}$} & Pre1 & $6.3 \pm 3.5$ & $6.0 \pm 2.5$ \\
\hline & Pre2 & $9.4 \pm 4.1$ & $8.5 \pm 3.6$ \\
\hline & Pre3 & $6.1 \pm 2.3$ & $5.8 \pm 2.3$ \\
\hline & Pre4 & $14.3 \pm 7.2$ & $13.9 \pm 6.2$ \\
\hline & Pre5 & $9.1 \pm 4.5$ & $7.6 \pm 4.0$ \\
\hline & Pre6 & $13.2 \pm 6.8$ & $13.0 \pm 5.5$ \\
\hline \multirow{6}{*}{$\mathrm{GRF}_{\mathrm{x}}[\mathrm{N}]$} & Pre1 & 20.5 & 22.1 \\
\hline & Pre2 & 33.1 & 22.7 \\
\hline & Pre3 & 25.1 & 25.6 \\
\hline & Pre4 & 20.7 & 17.2 \\
\hline & Pre5 & 31.5 & 23.6 \\
\hline & Pre6 & 28.7 & 24.3 \\
\hline \multirow{6}{*}{$\mathrm{GRF}_{\mathrm{y}}[\mathrm{N}]$} & Pre1 & 0.8 & 0.5 \\
\hline & Pre2 & 0.8 & 0.6 \\
\hline & Pre3 & 0.9 & 0.6 \\
\hline & Pre4 & 0.8 & 0.6 \\
\hline & Pre5 & 0.9 & 0.7 \\
\hline & Pre6 & 0.7 & 0.6 \\
\hline \multirow{6}{*}{$\mathrm{CoP}_{\mathrm{x}}[\mathrm{mm}]$} & Pre1 & 1.6 & 0.7 \\
\hline & Pre2 & 1.3 & 0.9 \\
\hline & Pre3 & 1.9 & 1.2 \\
\hline & Pre4 & 1.8 & 0.9 \\
\hline & Pre5 & 2.1 & 1.3 \\
\hline & Pre6 & 1.6 & 1.2 \\
\hline \multirow{6}{*}{$\begin{array}{l}\text { Human joint } \\
\text { moments }[\mathrm{Nm}]\end{array}$} & Pre1 & $7.7 \pm 4.0$ & $6.2 \pm 1.4$ \\
\hline & Pre2 & $10.1 \pm 4.7$ & $6.9 \pm 3.2$ \\
\hline & Pre3 & $9.1 \pm 4.8$ & $6.7 \pm 2.0$ \\
\hline & Pre4 & $9.1 \pm 4.7$ & $6.4 \pm 2.2$ \\
\hline & Pre5 & $10.7 \pm 4.2$ & $6.7 \pm 1.1$ \\
\hline & Pre6 & $9.6 \pm 5.5$ & $7.8 \pm 1.5$ \\
\hline \multirow{6}{*}{$\begin{array}{c}\text { Exoskeleton joint } \\
\text { moments }[\mathrm{Nm}]\end{array}$} & Pre1 & $6.0 \pm 4.6$ & $3.9 \pm 1.4$ \\
\hline & Pre2 & $6.8 \pm 5.6$ & $3.1 \pm 0.8$ \\
\hline & Pre3 & $7.3 \pm 6.3$ & $4.8 \pm 2.6$ \\
\hline & Pre4 & $5.7 \pm 4.5$ & $3.2 \pm 1.6$ \\
\hline & Pre5 & $6.7 \pm 6.2$ & $4.0 \pm 1.2$ \\
\hline & Pre6 & $5.7 \pm 5.1$ & $3.7 \pm 1.3$ \\
\hline \multirow{3}{*}{ Pelvis c.f. $[\mathrm{N}]$} & Pre1 & 9.7 & 38.4 \\
\hline & Pre2 & 11.9 & 33.8 \\
\hline & Pre3 & 13.1 & 38.9 \\
\hline \multirow{3}{*}{ Thigh c.f. $[\mathrm{N}]$} & Pre4 & 2.7 & 11.9 \\
\hline & Pre5 & 5.0 & 14.2 \\
\hline & Pre6 & 5.5 & 14.7 \\
\hline \multirow{3}{*}{ Shank c.f. $[\mathrm{N}]$} & Pre4 & 5.5 & 5.3 \\
\hline & Pre5 & 5.1 & 5.4 \\
\hline & Pre6 & 4.7 & 21.1 \\
\hline
\end{tabular}

$\mathrm{GRF}_{\mathrm{x}}$ and $\mathrm{GRF}_{\mathrm{y}}$ stand for horizontal and vertical ground reaction forces, $\mathrm{CoP}_{\mathrm{x}}$ for horizontal location of the centre of pressure, and c. f. for contact force. For joint coordinates, velocities and moments, mean \pm standard deviation across all joints are shown. Pre1 to Pre6 stand for prediction trials 1 to 6 (Pre1 to Pre3 with pressure data at the pelvis and Pre4 to Pre6 at the thigh and shank).

trials accurately. Mean RMSD values for joint coordinates ranged between 1.0 and 3.3 degrees, and between 6.1 and 14.3 degrees/s for joint angular velocities. When perturbing the values of the subject-exoskeleton contact model by $40 \%$ (Phase C2), those RMSD values were quite similar (Table III, see one example in Fig. 6). The tracking of ground reaction forces and joint moments was slightly better in two of the three calibration trials in Phase C2 compared to Phase C1. 

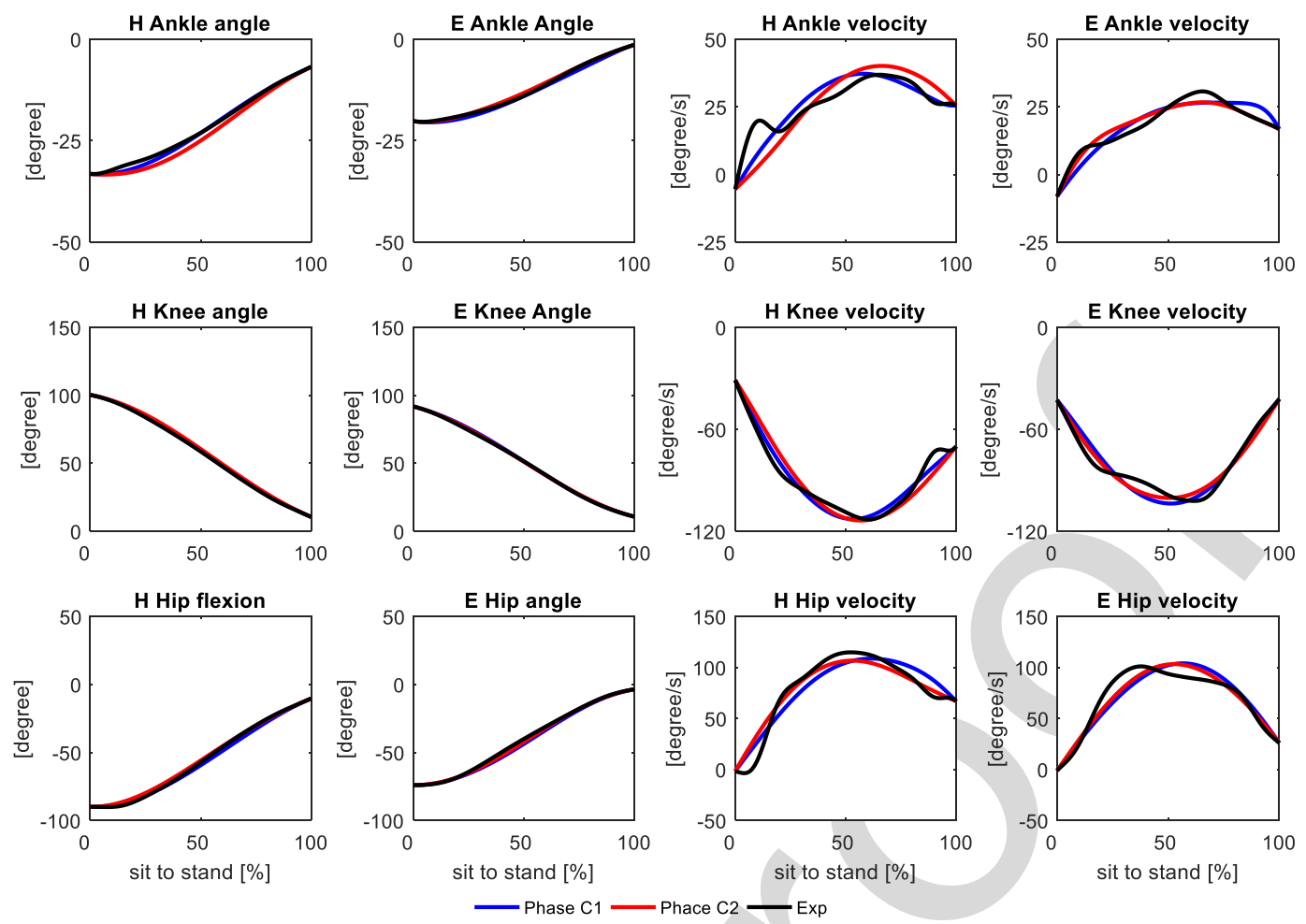

Fig. 6. Kinematics prediction of one prediction trial in Phases $\mathrm{C} 1$ and $\mathrm{C} 2$. Angles and angular velocities for ankle, knee and joint angles of the human $(\mathrm{H})$ and exoskeleton $(\mathrm{E})$ side. In blue, prediction with contact model values from Phases $\mathrm{A}$ and $\mathrm{B}$; in red with perturbed contact model values; in black, experimental data.
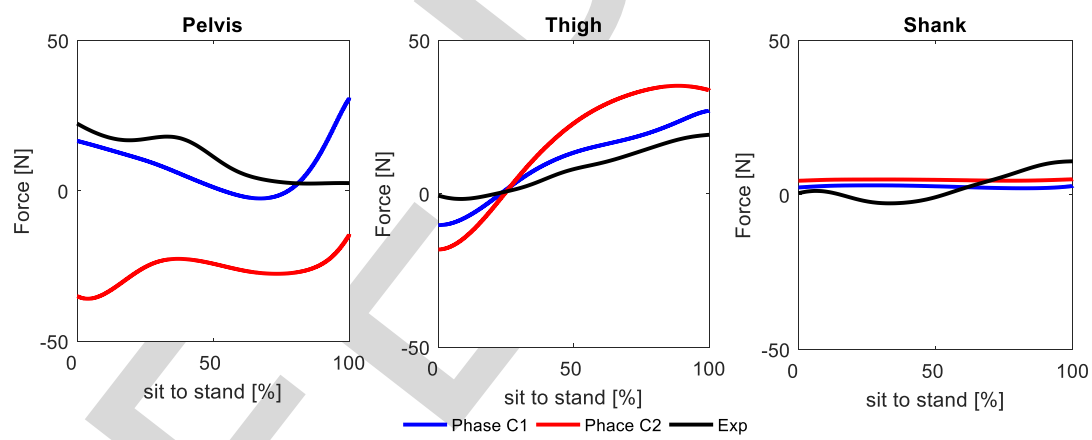

Fig. 7. Resultant subject-exoskeleton contact force predictions for two prediction trials in Phases $\mathrm{C} 1$ and $\mathrm{C} 2$. One trial was used to predict pelvis contact force and the other trial was used to predict thigh and shank contact forces. In blue, prediction with contact model values from Phases A and B: in red with perturbed contact model values; in black, experimental data.

In terms of RMSD values, the prediction of interaction forces was between 2 and 5 times better in Phase $\mathrm{C} 1$ than in Phase $\mathrm{C} 2$ at the pelvis and thigh, and 4.5 times better for one trial at the shank (Table III, see two examples in Fig. 7), which suggests that the calibration of subject-exoskeleton contact parameters had more influence on the contact force prediction than on the predicted movement.

\section{DISCUSSION}

This study aimed to calibrate foot-ground and subjectexoskeleton contact models to predict the collaborative movement and interaction forces between a subject and exoskeleton during sit-to-stand movements. We used experimental contact forces measured from pressure sensors and force plates from three sit-to-stand trials with the exoskeleton in passive mode to calibrate the models, and then we predicted the collaborative movement and their forces in three sit-tostand trials with the exoskeleton in assistance mode. The estimated contact parameter values allowed us to reproduce the experimental forces with the exoskeleton in passive mode (calibration trials) quite well, as well as the subject and exoskeleton kinematics.

Once the contact models were calibrated, the predicted movement with the exoskeleton in assistive mode (prediction trials) followed the experimental values (RMSD of angles <3.5 degrees, and RMSD of velocities < 15.0 degrees/s). In this case, the predicted subject-exoskeleton forces overall had the same magnitude as the experimental forces. We also predicted the movement and forces perturbing the subject-exoskeleton parameter values by $40 \%$. We observed that interaction force predictions diverged from experimental values, especially at the pelvis (the RMSD was 


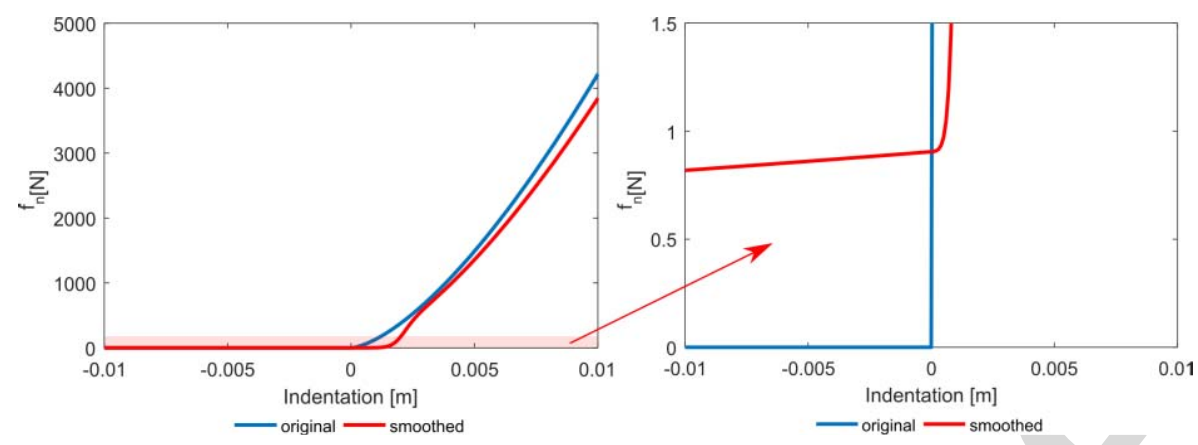

Fig. 8. Normal Hunt Crossley force as a function of the indentation. The zoom shows the non-zero slope of the curve at the shadowed area.

greater than $30 \mathrm{~N}$ ) and the thigh (RMSD of contact forces $>$ $10 \mathrm{~N}$ ) (Fig. 7). Therefore, once the parameter values of an initial prototype have been calibrated, the proposed method will be useful to predict optimal movements (e.g. with the criterion to minimize contact forces to improve comfort), or to analyze how the contact forces would change when modifying the control of the exoskeleton or when modifying the stiffness of one part, with no need to reproduce all movements experimentally.

Some limitations were identified in this study. First, we used a torque-driven planar model. Although the model accounted for the dominant forces and moments (produced in the sagittal plane during sit-to stand movements), it would also be valuable to explore the effect in the other planes, such as hip adduction and rotation. A muscle-driven model may also lead to more realistic kinematic and dynamic results than a torque-driven model [24], [25]. Second, we had experimental limitations, since we could not have information of shear forces, which may give important interaction information. Another sensor system to measure shear forces should be used since the forces in this direction are also considered to produce discomfort [26], [27]. Third, we only predicted sit-tostand movements in one subject and those movements were similar to the ones used for calibrating the contact models. The method could also be applied to other types of movements and subjects to assess the validity of the calibrated models for other movements.

In conclusion, our simulation framework can predict realistic kinematics and forces with proper calibration of contact models; we observed that, without calibration, contact forces may not be realistic. These results reinforce the importance of validating the results obtained with musculoskeletal models [7]. Future directions include predicting three dimensional movements and other types of movements, such as walking.

\section{APPENDIX 1: SMOOTH FOOT-GROUND CONTACT MODEL}

We used a smooth foot-ground (spheres-plane) contact model based on the original version of the Hunt Crossley contact model in Simbody [18] to avoid the optimizer (based on gradient based methods) to fall in a region with discontinuities. The main two expressions that we modified were related to the normal force and the Stribeck function that computes the friction coefficient. To compute the normal force, Simbody uses the following expressions:

$$
\begin{aligned}
& f_{p}=x^{\frac{3}{2}} \\
& f_{v}=1+1.5 c \dot{x} \\
& f_{n}=\frac{4}{3} k^{\frac{3}{2}} \sqrt{r} f_{p} f_{v}
\end{aligned}
$$

where $x$ is the indentation, $\dot{x}$ is the indentation velocity, $c$ is the damping coefficient, $k$ is the stiffness, $r$ is the radius of the sphere, $f_{p}$ and $f_{v}$ are terms dependent on indentation and its velocity, respectively, and $f_{n}$ is the normal force. We multiplied $f_{p}$ and $f_{v}$ by terms to avoid negative contact force values and ensure the functions are continuously differentiable:

$$
\begin{aligned}
& f_{\text {p nonneg }}=f_{p}\left(\frac{1}{2}+\frac{1}{2} \tanh \left(b_{c} x\right)\right) \\
& f_{v \text { nonneg }}=f_{v}\left(\frac{1}{2}+\frac{1}{2} \tanh \left(b_{c}\left(\dot{x}+\frac{2}{3 c}\right)\right)\right)
\end{aligned}
$$

Then, following Equation 3, we obtained a new expression for the normal force. We also included a term to avoid a zero slope in the contact force when there is no actual contact:

$$
\begin{aligned}
f_{\text {slope }}=e^{\frac{x-0.01}{0.1}} b_{n}\left(\frac{1}{2}\right. & \left.+\frac{1}{2} \tanh \left(b_{d} x\right)\right) \\
& \times\left(\frac{1}{2}+\frac{1}{2} \tanh \left(b_{v}\left(\dot{x}+\frac{2}{3 c}\right)\right)\right)
\end{aligned}
$$

where $b_{c}, b_{d}, b_{n}$ and $b_{v}$ were constant parameters. Since we are using gradient-based methods to solve the optimization problem, the use of this term allows a non-zero gradient value even when the foot does not penetrate the ground. The added force when out of contact is negligible (lower than $1 \mathrm{~N}$ ).

Then, we combined all terms to avoid discontinuities and ensure smoothness in the stick-to-slip transition (see Fig. 8 for an example of the smoothness and non-zero slope).

Regarding the friction coefficient, the original curve of the Stribeck function, which is not smooth, can be divided into terms that depend on viscous friction $\left(\mu_{1}\right)$ and into terms that do not $\left(\mu_{2}\right): \mu\left(v_{\text {rel }}\right)=\mu_{1}\left(v_{\text {rel }}\right)+\mu_{2}\left(v_{\text {rel }}\right)$.

We approximated $\mu_{2}$ with a three-part function:

$$
\begin{aligned}
\mu_{2}\left(v_{\text {rel }}\right) & = \begin{cases}u_{d} & v_{\text {rel }}>3 \\
u_{s}-\left(u_{s}-u_{d}\right) \text { step } 5\left(\frac{v_{\text {rel }}-1}{2}\right) & 1<=v_{\text {rel }}<3 \\
u_{s} \text { step } 5\left(v_{\text {rel }}\right) & v_{\text {rel }}>1\end{cases}
\end{aligned}
$$




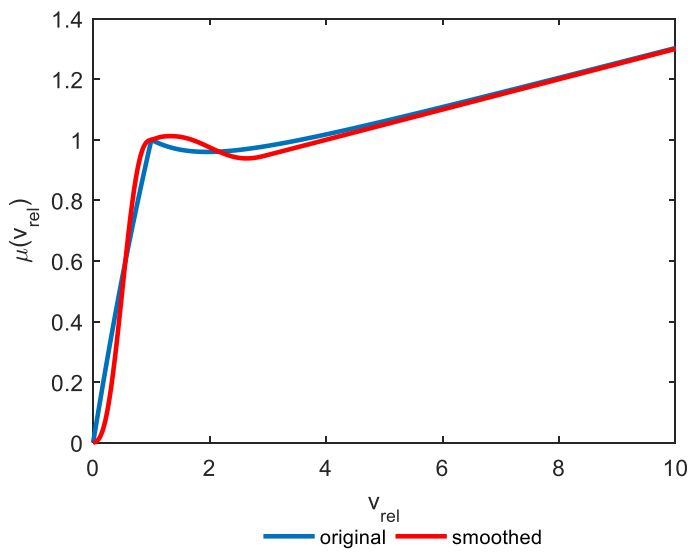

Fig. 9. Friction coefficient curve as a function of $\mathrm{v}_{\mathrm{rel}}$.

where $v_{\text {rel }}=v_{\text {slip }} / v_{t}, v_{\text {slip }}$ is the module of the tangential velocity of the contact point with respect to ground, $u_{s}$ and $u_{d}$ are the static and dynamic friction coefficients, $v_{t}$ is the transition velocity, and step 5 is the approximation of the step function with a $5^{\text {th }}$ order polynomial. We used a single expression to represent $\mu\left(v_{r e l}\right)$, smoothing the transitions between regions (see Fig. 9).

\section{ACKNOWLEDGMENT}

The authors thank A. Bruijnes and L. Muraru for data collection.

\section{REFERENCES}

[1] A. M. Dollar and H. Herr, "Lower extremity exoskeletons and active orthoses: Challenges and state-of-the-art," IEEE Trans. Robot., vol. 24, no. 1, pp. 144-158, Feb. 2008.

[2] T. Yan, M. Cempini, C. M. Oddo, and N. Vitiello, "Review of assistive strategies in powered lower-limb orthoses and exoskeletons," Robot. Auton. Syst., vol. 64, pp. 120-136, Feb. 2015.

[3] J. L. Pons, Wearable Robots: Biomechatronic Exoskeletons. London, U.K.: Wiley, 2008.

[4] J. E. Grey, S. Enoch, and K. G. Harding, "ABC of wound healing: Venous and arterial leg ulcers," BMJ, vol. 332, pp. 472-475, Apr. 2006.

[5] Y. Li, S.-H. Chang, G. Francisco, and H. Su, "Interaction force modeling for joint misalignment minimization toward bio-inspired knee exoskeleton design," in Proc. Des. Med. Devices Conf., Apr. 2018, pp. 1-3.

[6] A. J. Young and D. P. Ferris, "State of the art and future directions for lower limb robotic exoskeletons," IEEE Trans. Neural Syst. Rehabil. Eng., vol. 25, no. 2, pp. 171-182, Feb. 2017.

[7] J. L. Hicks, T. K. Uchida, A. Seth, A. Rajagopal, and S. L. Delp, "Is my model good enough? best practices for verification and validation of musculoskeletal models and simulations of movement," J. Biomech. Eng., vol. 137, no. 2, Feb. 2015, Art. no. 020905.

[8] J. Zhang et al., "Human-in-the-loop optimization of exoskeleton assistance during walking," Science, vol. 356, no. 6344, pp. 1280-1283, Jun. 2017.

[9] M. Millard, M. Sreenivasa, and K. Mombaur, "Predicting the motions and forces of wearable robotic systems using optimal control," Front. Robot. AI, vol. 4, pp. 1-12, Aug. 2017.
[10] P. Manns, M. Sreenivasa, M. Millard, and K. Mombaur, "Motion optimization and parameter identification for a human and lower back exoskeleton model," IEEE Robot. Autom. Lett., vol. 2, no. 3, pp. 1564-1570, Jul. 2017.

[11] M. Donati et al., "A flexible sensor technology for the distributed measurement of interaction pressure," Sensors, vol. 13, no. 1 , pp. 1021-1045, Jan. 2013.

[12] S. M. M. de Rossi et al., "Sensing pressure distribution on a lower-limb exoskeleton physical human-machine interface," Sensors, vol. 11, no. 1, pp. 207-227, Dec. 2011.

[13] K. Tanghe et al., "Predicting seat-off and detecting start-of-assistance events for assisting sit-to-stand with an exoskeleton," IEEE Robot. Autom. Lett., vol. 1, no. 2, pp. 792-799, Jul. 2016.

[14] K. Junius et al., "Mechatronic design of a sit-to-stance exoskeleton," in Proc. 5th IEEE RAS/EMBS Int. Conf. Biomed. Robot. Biomechatron., Aug. 2014, pp. 945-950.

[15] J. Vantilt, E. Aertbeliën, F. De Groote, and J. De Schutter, "Optimal excitation and identification of the dynamic model of robotic systems with compliant actuators," in Proc. IEEE Int. Conf. Robot. Autom., May 2015, pp. 2117-2124.

[16] V. Grosu, C. R. Guerrero, B. Brackx, S. Grosu, B. Vanderborght, and D. Lefeber, "Instrumenting complex exoskeletons for improved humanrobot interaction," IEEE Instrum. Meas. Mag., vol. 18, no. 5, pp. 5-10, Oct. 2015.

[17] A. Seth et al., "OpenSim: Simulating musculoskeletal dynamics and neuromuscular control to study human and animal movement," PLoS Comput. Biol., vol. 14, no. 7, pp. 1-20, Jul. 2018.

[18] M. A. Sherman, A. Seth, and S. L. Delp, "Simbody: Multibody dynamics for biomedical research," Procedia IUTAM, vol. 2, pp. 241-261, Jan. 2011

[19] A. J. Van Den Bogert, D. Blana, and D. Heinrich, "Implicit methods for efficient musculoskeletal simulation and optimal control," Procedia IUTAM, vol. 2, pp. 297-316, Jan. 2011.

[20] J. A. E. Andersson, J. Gillis, G. Horn, J. B. Rawlings, and M Diehl, "CasADi: A software framework for nonlinear optimization and optimal control," Math. Program. Comput., vol. 11, no. 1, pp. 1-36, Mar. 2018.

[21] A. Wächter and L. T. Biegler, "On the implementation of an interiorpoint filter line-search algorithm for large-scale nonlinear programming," Math Progr., vol. 106, no. 1, pp. 25-57, Mar. 2006.

[22] P. M. J. Van Den Hof, J. F. M. Van den Doren, and S. G. Douma, "Identification of Parameters in Large Scale Physical Model Structures, for the purpose of model-based operations," Model. Control Bridg. Rigorous Theory Adv. Technol., vol. 125, pp. 125-143, Jul. 2009.

[23] K. J. Kuchenbecker, J. G. Park, K. Kuchenbecker, J. Park, and G. Niemeyer, "Characterizing the human wrist for improved haptic interaction," in Proc. IMECE, 2003, pp. 591-598.

[24] F. De Groote, A. L. Kinney, A. V. Rao, and B. J. Fregly, "Evaluation of direct collocation optimal control problem formulations for solving the muscle redundancy problem," Ann. Biomed. Eng., vol. 44, no. 10, pp. 2922-2936, Oct. 2016

[25] A. Rajagopal, C. L. Dembia, M. S. DeMers, D. D. Delp, J. L. Hicks, and S. L. Delp, "Full-body musculoskeletal model for muscle-driven simulation of human gait," IEEE Trans. Biomed. Eng., vol. 63, no. 10 , pp. 2068-2079, Oct. 2016.

[26] E. Boutwell, R. Stine, A. Hansen, K. Tucker, and S. Gard, "Effect of prosthetic gel liner thickness on gait biomechanics and pressure distribution within the transtibial socket," J. Rehabil. Res. Dev., vol. 49, no. 2, pp. 227-240, Feb. 2012.

[27] K. A. Witte, J. Zhang, R. W. Jackson, and S. H. Collins, "Design of two lightweight, high-bandwidth torque-controlled ankle exoskeletons," in Proc. IEEE Int. Conf. Robot. Autom., May 2015, pp. $1223-1228$ 